\title{
Reactivity of chitosan derivatives and their interaction with guanine: A computational study
}

\author{
BHABESH CHANDRA DEKA and PRADIP KR BHATTACHARYYA* \\ Department of Chemistry, Arya Vidyapeeth College, Guwahati 781 016, India \\ e-mail: prdpbhatta@yahoo.com
}

MS received 2 December 2015; revised 30 January 2016; accepted 16 February 2016

\begin{abstract}
The present study delves into the reactivity of a few chitosan derivatives (CSDs) and their interaction with guanine in vacuum and in different phases. Increase in the polarity of the solvent lowers reactivity of the chosen derivatives (evaluated by using reactivity descriptors). Interaction between the CSDs and guanine (measured by interaction energy) weakens in solvent media and CSD-guanine interaction is weaker than the interaction between guanine and unmodified chitosan (CS). Chemical stability of CSD-guanine adducts remains similar to that of CS-guanine adduct in both polar and non-polar media. Moreover, CSD-guanine adducts exhibit comparable thermodynamic stability (quantified by free energy of solvation, $\Delta \mathrm{G}_{\text {sol }}$ ) to that of unmodified CS-guanine adduct in non-polar solvent but in polar medium they are immensely destabilized in comparison to CS-guanine adduct. Observed theoretical results are expected to provide guidance for future relevant experimental research on gene delivery by CS derivatives.
\end{abstract}

Keywords. Density functional theory; hydrogen bonding; chitosan derivative; guanine; solvent effect

\section{Introduction}

Chitosan (CS) is a biodegradable and biocompatible polymeric glucosamine derived from chitin. Mumper et al., first described CS as a delivery system for plasmids enumerating its strong nucleic acid (NA) binding ability in $1998 .{ }^{1}$ Ever since, CS has been one of the most extensively studied non-viral carrier of DNA and small interfering RNA (siRNA). ${ }^{2-6}$ However, several demerits of CS, such as poor solubility in physiological $\mathrm{pH}$, low transfection efficiency, mistimed dissociation of CS-NA complex and low cell specificity have posed stiff challenges in formulating CS-aided gene delivery systems. ${ }^{7}$ Chemical modification of CS has been employed as a tool to circumvent these limitations. Occurrence of an amino functionality at 2position of the six membered ring lends itself amenable for structural alterations ${ }^{8}$ and recent literature reports insightful developments taking place with respect to a number of tailor-made chitosan derivatives (CSDs). ${ }^{9}$ Some important modes of chemical modification vis $a$ vis their objectivity are: (i) quarternisation is used to increase solubility, in order to keep the amino group in $\mathrm{CS}$ remain protonated at physiological $\mathrm{pH}$ so as to maintain the stability of the CS-plasmid complex with enhanced muco-adhesive character; ${ }^{10,11}$ (ii) N-Acylation increases hydrophobic character for

*For correspondence enhanced trans-membrane permittivity; ${ }^{12}$ (iii) thiolation improves muco-adhesive, permeation and controlled drug release properties; ${ }^{13}$ (iv) improvement in transfection efficiency is achieved by alkylation; ${ }^{14,15}$ (v) hydrophilicity and controlled release of the drug is improved by glycol conjugation; ${ }^{16}$ (vi) hydroxyalkylation is used to introduce amphiphilic character and for stabilization of the drug-carrier complex. ${ }^{17-19}$

In complex matrices like a cell, the NA moiety may undergo degradation due to attack by serum, histones or other ions. Especially, the hetero-atoms in the nucleobase moiety in the CSD-nucleobase adduct are vulnerable to electrophilic attack by the cellular ions. ${ }^{20,21}$ Stability as well as reactivity of the CSD and CSD-NA complex (polyplex) plays an important role in determining the intact NA to be received by the nucleus of the target cell as well as the fate of the CSD after transferring NA to the target cell. In addition, strength of interaction between CSDs and NA is also crucial in binding as well as release of the NA from the CSD-NA polyplex. A thorough understanding on the stability as well as reactivity of CSDs, CSD-NA adducts and the strength of interaction between CSDs and nucleobases is deemed essential in terms of evaluating the efficacy of a particular CSD as NA carrier.

The present study is an attempt to explore the reactivity of a group of CSDs and their interaction with nucleobases in DNA/RNA. Herein, eight CSDs covering the 
aforementioned modes of modification are considered. These include, trimethyl chitosan (TMC) (1) N-(2-hydroxyl) propyl-3-trimethylammonium chitosan (HTC), (2) CS-2-iminothiolane (3) CS-cysteine, (4) Acetyl CS, (5) N- octyl CS, (6) O-glycol CS and (7) N- octyl O-glycol CS (8). As far as mode of interaction between CSD and nucleobases is concerned, a number of earlier reports offer insightful studies describing primary electrostatic interaction of CS and its derivatives with the backbone of the nucleic acid. ${ }^{1,3,8}$ However, the exact mechanistic detail regarding the binding of nucleic acid at the laboratory bench and its release inside the cytoplasm by CS and its derivatives has not yet been achieved. Some related studies show that a secondary interaction such as hydrogen bonding between protonated CS/CSDs with hetero centers of nucleobases plays an important role in binding and release of NA during gene delivery. Especially an earlier investigation by Mao et al., outlined a crucial role of hydrogen bonding in binding of DNA/RNA by CS. ${ }^{22}$ Furthermore, our recent work also enumerates the role of hydrogen bonding in formation and dissociation of CS-nucleobase adducts. ${ }^{23}$ In line with our earlier work, the present study focuses on the secondary interaction i.e., hydrogen bonding and not on the primary interaction with phosphate backbone. In view of the complex physiological medium (from a non-polar cellmembrane to polar cytoplasm) encountered by CSDNA complex, we have also examined the impact of polarity of solvent on interaction energy and reactivity parameters of the CSD-nucleobase complex taking three solvents, namely carbon tetrachloride, ethanol and water into considerations.

\section{Theoretical and computational details}

Density functional theory (DFT) and density functional reactivity theory (DFRT) were used in this study. These are acclaimed by several research groups as powerful tools to unravel many intricate processes at atomic/ molecular level. ${ }^{24-30}$ Usually, all the heteroatoms in a nucleobase are prone to electrophilic attack,,$^{31,32}$ and the order of reactivity of the nucleophilic sites of different nucleobases is reported as: guanine N7 > adenine $\mathrm{N} 1>$ cytosine $\mathrm{N} 1>$ adenine N3 in RNA and guanine N7 $>$ adenine N3 > cytosine N1 in DNA. ${ }^{33,34}$ Furthermore, our recent studies also establish a stronger interaction between protonated CS and guanine than with other nucleobases. ${ }^{23,35}$ There are two possible modes that protonated CSDs may interact with guanine; mode a- formation of two hydrogen bonds with N7 and O6 centres and mode $\mathrm{b}$ - formation of a single hydrogen bond at N3 centre. The isosurface diagram of guanine (figure 2) depicts a negative charge density (indicated by red region) spreading only over the N7 and O6 centers which points at a greater inclination of these two centers than other electronegative centers (including N3) towards electro-positive sites. In our recent work, we studied both the possibilities in adduct formation between chitosan and guanine where it was observed that in the former mode (two $\mathrm{H}$ bonds at $\mathrm{N} 7$ and $\mathrm{O} 6$ of guanine), interaction energies were -40.67 , $-35.61,-19.88$ and $-18.75 \mathrm{kcal} / \mathrm{mol}$ in gas, cyclohexane, ethanol and water media, respectively, while interaction energy values concomitant with the later mode (hydrogen bond at $\mathrm{N} 3$ centre of guanine) were considerably low, $-24.08,-15.77,-10.63$, and $-9.90 \mathrm{kcal} / \mathrm{mol}$ in the respective media. ${ }^{35}$ It shows a stronger interaction (and thus greater stability of adduct) in case of the mode "a" as compared to "b" mode. In view of these facts, while modeling the association geometries we have chosen guanine among the five nucleobases as a representative one and the mode "a" (i.e., formation of two hydrogen bonds with N7 and O6 centers) is considered only. The sugar (ribose) phosphate part of the nucleotides is replaced by a methyl group for the sake of computational simplicity. Among various DFT functionals, several recent studies have outlined the suitability of CAM-B3LYP for estimating strength of hydrogen bonding interaction. ${ }^{36,37}$ CAM-B3LYP is an exchangecorrelation energy functional based on the Coulombattenuating method (CAM), which combines the hybrid qualities of Becke three parameter exchange and Lee, Yang and Parr correlation functional (B3LYP) and the dispersion correction essential for calculating interaction energy of hydrogen bonded systems. ${ }^{38,39}$ Geometrical minima of chitosan, guanine, the chosen derivatives and the adducts are obtained by using $6-31++\mathrm{G}$ $(\mathrm{d}, \mathrm{p})$ basis set and are ascertained by the absence of any imaginary frequencies.

Chemical stability of a molecular system is explained on the basis of reactivity descriptors provided within the framework of DFRT. Global hardness, (also called chemical hardness), chemical potential, global electrophilicity, etc., are grouped into global reactivity descriptors, while fukui functions (FF), local electrophilicity, local softness, etc., are categorized as local reactivity descriptors. ${ }^{40}$ Chattaraj and co-workers proposed the group philicity $\left(\omega_{g}\right)$ as the summed condensed philicity of atoms present in a group as a measure of propensity of a particular group to undergo an electrophilic/nucleophilic/radical attack. ${ }^{41}$ Several research groups have tested the efficiency of these descriptors in rationalizing the reactivity patterns of many molecular and biomolecular systems. ${ }^{42-47} \mathrm{Pal}$ and 
co-workers have studied the behavior of these descriptors in presence of solvent media. ${ }^{48}$

DFRT defines chemical potential $(\mu)$ and global hardness $(\eta)$ as the first and second derivative of energy with respect to the number of electrons. ${ }^{49,50}$ Use of finite difference approximation and Koopmans' theorem ${ }^{51}$ leads to the working formulae for $\mu$ and $\eta$ as:

$$
\begin{aligned}
& \eta=\left(\varepsilon_{L U M O}-\varepsilon_{\text {НомO }}\right) / 2 \\
& \mu=\left(\varepsilon_{\text {LUMO }}+\varepsilon_{\text {HOMO }}\right) / 2
\end{aligned}
$$

Electrophilicity $(\omega)$ is expressed as: ${ }^{52}$

$$
\omega=\mu^{2} / 2 \eta
$$

The inherent propensity of positively charged amine group in CSDs for hydrogen bonding with heterocentres of nucleobases depends on its electrophilic character which can be quantified by nucleophilic fukui function $\left(f^{+}\right)$of the $\mathrm{N}$ atom, and more precisely by group philicity $\left(\omega_{g}^{+}\right)$of amine group in CSDs. Accordingly, we have examined the reactivity of the amine group in terms of the $f^{+}$of the $\mathrm{N}$ atom, and the $\omega_{g}^{+}$of amine group in CSDs.

For an atom ' $x$ ' in a molecule with $N$ electrons, local electrophilicity index $\left(\omega_{x}^{+}\right)$for nucleophilic attack is defined as: ${ }^{51}$

$$
\omega_{x}^{+}=\omega f_{x}^{+}
$$

where, $f_{x}^{+}$is the nucleophilic fukui function (FF) and is calculated by using-

$$
f_{x}^{+}=\left[\rho_{x}\left(\mathrm{~N}_{0}+1\right)-\rho_{x}\left(\mathrm{~N}_{0}\right)\right]
$$

where, $\rho_{x}\left(\mathrm{~N}_{0}\right)$ and $\rho_{x}\left(\mathrm{~N}_{0}+1\right)$ are electronic population on atom $x$ in the molecule with $\mathrm{N}_{0}$, and $\mathrm{N}_{0}+1$ electrons respectively. ${ }^{53}$ The most electrophilic site in a molecule is the one possessing the maximum $f_{x}^{+}$. FF in both gas and solvent media for the chosen CSDs is computed by using Hirshfeld's population scheme employing the same CAMB3lyp 6-31G++(d.p) level of theory. ${ }^{54}$ Roy et al., have thoroughly analysed the success of this scheme. ${ }^{55,56}$ Group philicity $\left(\omega_{g}^{+}\right)$is the condensed philicity summed over a group of surrounding atoms of $\mathrm{N}$ of amine group of $\mathrm{CSD}^{57}$ and is expressed as:

$$
\omega_{g}^{+}=\sum_{x=1}^{n} \omega_{x}^{+}
$$

The interaction energy $\left(\Delta \mathrm{E}_{\mathrm{int}}\right)$ between CSD and guanine is calculated using super-molecular approach, $\left[\Delta \mathrm{E}_{\text {int }}=\left(\mathrm{E}_{\mathrm{CSD} \text {-gua }}\right)-\left(\mathrm{E}_{\mathrm{CSD}}+\mathrm{E}_{\text {gua }}\right)\right]$, where $\mathrm{E}_{\mathrm{CSD} \text {-gua }}$ is the energy of adduct, $\mathrm{E}_{\mathrm{CSD}}$ is the energy of the chitosan derivative and $\mathrm{E}_{\text {gua }}$ is the energy of the guanine molecule. In calculating the interaction energies we have included the basis set superposition error (BSSE) correction using counterpoise $=\mathrm{N}^{58}$
Out of different models for accounting the solvation energies, dielectric continuum solvation models have been widely and successfully applied. In the selfconsistent versions of the continuum, the solvent is considered as a continuous dielectric medium that is polarized by the solute. This leads to a reaction field that polarizes the solute, which changes the solvent polarization leading to a self-consistent reaction field (SCRF). Polarizability continuum model (PCM) is a popular way to implement the SCRF approach which describes the solvent polarization in terms of the electrostatic potential. ${ }^{59}$ The PCM assumes that all solute charge density resides inside the cavity. This model was developed by Tomasi's group and subsequently a wealth of documented literature shows its application in different molecular systems. ${ }^{60}$

Furthermore, with an aim to predict the thermodynamic stability of the CSD-nucleobase adduct in the solvent media, free energy of solvation $\left(\Delta \mathrm{G}_{\mathrm{sol}}\right)$ of adducts was calculated by using the SMD keyword, as implemented in Gaussian09, which does an IEFPCM calculation with radii and non-electrostatic terms for Truhlar and coworkers' SMD solvation model. ${ }^{61}$

All calculations are performed by using Gaussian $09 .{ }^{62}$

\section{Results and Discussion}

\subsection{Stability/Reactivity of the CSD}

Global hardness $(\eta)$ encompasses contribution of energy of both HOMO and LUMO and is one of the most preferred reactivity descriptors by researchers in interpretation of chemical stability of a system. As such, $\eta$ of the chosen CSDs is calculated to evaluate their chemical stability in different phases. Moreover, vulnerability of a system for electrophilic, nucleophilic or radical attack at a particular atom or group (local reactivity) is explained on the basis of group philicity $\left(\omega_{g}\right)$. Herein, propensity of the protonated amine group of CSDs for hydrogen bonding with guanine is evaluated by calculating group philicity for nucleophilic attack $\left(\omega_{g}^{+}\right)$.

3.1a Global hardness ( $\eta$ ) of CSDs: A higher value of $\eta$ suggests greater chemical stability of a molecule (according to maximum hardness principle, MHP). ${ }^{63}$ A comparison of global hardness values of the chosen derivatives and unmodified CS in different phases is shown in figure 1 .

Gas phase stability order of the chosen CSDs is- CS $<$ CS-2-iminothiolane $<$ CS-cysteine $<$ O-glycol CS $<$ acetyl $\mathrm{CS} \approx \mathrm{HTC}<\mathrm{N}$-octyl O-glycol CS $<\mathrm{N}$-octyl 


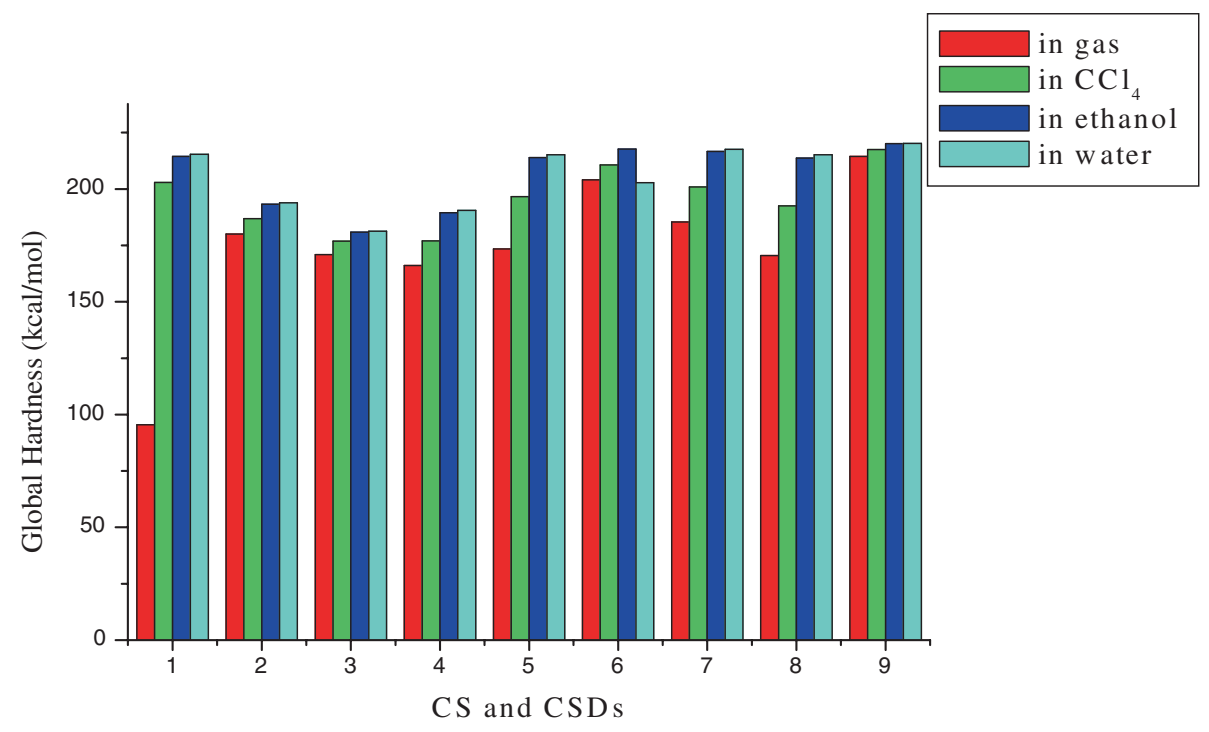

Figure 1. Global hardness $(\mathrm{kcal} / \mathrm{mol})$ of chitosan and chitosan derivatives in different media (in $\mathrm{kcal} / \mathrm{mol}$ ) at CAMB3LYP/6-31++G(d,p) level of theory. $1 \mathrm{CS}, 2$ acetyl CS, 3 CS-cysteine, 4 CS-2-iminothiolane, 5 HTC 6 N-octyl CS, 7 N-octyl O-glycol $\mathrm{CS}, 8 \mathrm{O}-$ glycol CS and 9 TMC.

CS < TMC. Maximum stability of TMC may be due to compensation of positive charge density over amine nitrogen by the three methyl groups attached with it. In solvent phase, stability of the CSDs increases and the polarity of the solvent contribute to the stability; greater stability in aqueous phase than that in carbon tetrachloride is observed. In carbon tetrachloride phase the stability order is- CS-2iminothiolane $<$ CS-cysteine $<$ acetyl CS $<$ O-glycol CS $<$ HTC $<$ N-octyl O-glycol $\mathrm{CS}<\mathrm{CS}<\mathrm{N}$-octyl CS $<$ TMC. Aqueous phase stability of the CSDs follows the order- CS-cysteine $<$ CS2-iminothiolane $<$ acetyl CS $<$ O-glycol CS $<\mathrm{N}$-octyl O-glycol CS $<$ HTC $<$ CS $<$ TMC. Thus, the observed trend indicates that the effect exerted by solvent polarity on chemical stability of CSDs is not uniform, an observation that resembles with earlier study on reactivity of a few cationic drug intermediates. ${ }^{64} \mathrm{~N}$-octyl $\mathrm{CS}$ is a bit less stable in aqueous phase than in $\mathrm{CCl}_{4}$ and ethanol medium. This may be due to hydrophobic interaction of its alkyl chain. Earlier too, stability of a few drug molecules is reported to increase in aqueous medium ${ }^{65}$ and results obtained in this study are in qualitative agreement with it.

3.1b Group philicity of amine group $\left(\omega_{g}^{+}\right)$: Involvement of the amine group of CS in formation of hydrogen bonding with nucleobases is enumerated in recent studies. ${ }^{23}$ It is demonstrated that the $\mathrm{CS}-\mathrm{NH}_{3}^{+}$group acts as $\mathrm{H}$-donor and the nucleobases act as $\mathrm{H}$ acceptor. As the transfer of proton/electrons between CSD and guanine is the crux of the interaction, electrophilicity, a local reactivity descriptor can be utilized to understand the adduct formation. According to the generalized concept of philicity, the preferred site for nucleophilic attack in a molecule is the one for which $\omega_{g}^{+}$(group philicity for nucleophilic attack) value is maximum. ${ }^{66}$ $\mathrm{H}$ - donating ability of a CSD (which is also proportional to the $\omega_{g}^{+}$value of amine group) is expected to be influenced by the nature of functional modification and the molecular environment as well. Therefore, group philicity of the amine group $\left(\omega_{g}^{+}\right)$in the chosen derivatives in different media is calculated and is presented in table 1.

Values in table 1, suggest that the amine group of the chosen CSDs exhibits higher value of $\omega_{g}^{+}$in gas phase than in solvent phase. Increase in di-electric constant of the medium expectedly lowers $\omega_{g}^{+}$in all the chosen adducts. This observation predicts weakening of interaction between CSDs and guanine with increasing polarity of the solvent. The solvent continuum around the solute molecules is reported to influence the local reactivity quantities such as the philicity indices wherein, it is shown that solvation effect reduces the intrinsic chemical reactivity at particular sites in selected organic molecules towards nucleophilic attack. ${ }^{67}$ Result obtained in this investigation also shows consistency with literature.

\subsection{Adduct formation between CSD and guanine}

Numerous experimental and theoretical investigations have characterized hydrogen bonding interactions in both solid and liquid states of anhydrous polymorph of chitosan and its derivatives. ${ }^{68-70}$ Under conditions 
Table 1. Group philicity of amine group of CS and CSDs.

\begin{tabular}{lcccc}
\hline CSD & $\omega_{g}^{+}$(gas) in kcal/mol & $\omega_{g}^{+}\left(\mathrm{CCl}_{4}\right)$ in kcal $/ \mathrm{mol}$ & $\omega_{g}^{+}$(ethanol) in kcal/mol & $\omega_{g}^{+}\left(\mathrm{H}_{2} \mathrm{O}\right)$ in kcal $/ \mathrm{mol}$ \\
\hline CS & 7.55 & 2.50 & 1.65 & 1.60 \\
TMC & 6.19 & 4.05 & 2.82 & 2.56 \\
HTC & 2.59 & 1.57 & 1.83 & 1.38 \\
CS-2iminothiolane & 1.85 & 1.18 & 1.01 & 0.81 \\
CS-cysteine & 2.64 & 1.75 & 1.17 & 0.88 \\
acetyl CS & 2.99 & 1.95 & 1.30 & 1.26 \\
N-octyl CS & 3.30 & 2.20 & 1.49 & 1.55 \\
O-glycol CS & 4.70 & 3.29 & 2.34 & 2.26 \\
N-octyl O-glycol CS & 2.68 & 1.95 & 1.09 & 1.00 \\
\hline
\end{tabular}

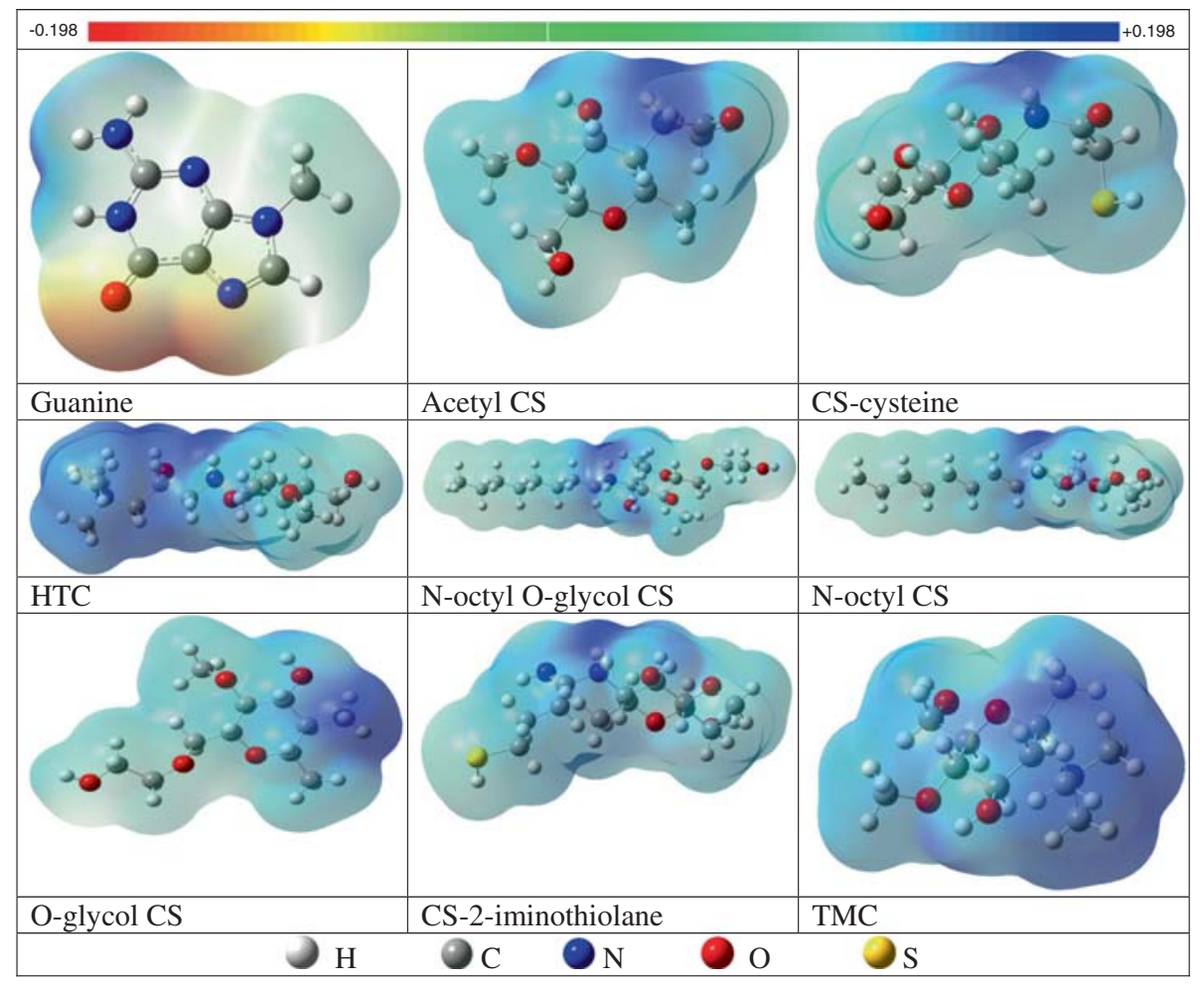

Figure 2. Isosurface diagrams of chitosan and chitosan derivatives (with 0.001 au density).

where CS is slightly charged, hydrogen bonding is responsible for binding between CS and nucleic acids. ${ }^{22}$ A critical role of hydrogen bonding in adduct formation between CS and nucleobases is also previously enlightened. ${ }^{23}$ Herein, a theoretical evidence of hydrogen bonding between CSD and nucleobases focusing on guanine as a representative case is pursued.

Hydrogen bonding ability of the amine hydrogen atoms in various CSDs can be predicted by their group charge distribution (NBO charges). The calculated NBO charges of the amine $\mathrm{H}$-atom of different CSDs is calculated to be approximately 0.48 au per $\mathrm{H}$ - atom which hints at the ability of these amine groups to act as $\mathrm{H}$ - donor during hydrogen bond formation.

Moreover, electrostatic interaction between the nucleophilic sites of guanine and amine $\mathrm{H}$ centres or other possible electrophilic sites in CSD can be understood from the electrostatic potential in an outer surface of the molecules. Figure 2 presents the iso surface diagram (with 0.001 au density) of CSDs and guanine which depicts a negative charge (red) region around the N7 and the $\mathrm{O} 6$ centers of guanine (acting as $\mathrm{H}$-acceptor) and a positive charge density (blue region) around amine $\mathrm{H}$ - centers of the considered CSDs as well as - $\mathrm{OH}$ functional group (acting as $\mathrm{H}$ - donor) in HTC and glycolated CSDs (7 and 8).

The gas phase optimized structures of the chosen CSD-gua adducts together with the hydrogen bond lengths $\left(\mathrm{r}_{\mathrm{hb}}\right)$ are shown in figure 3 . Except in case of TMC-gua, distance between amine hydrogen and $\mathrm{O6}$ or $\mathrm{N} 7$ of guanine in the considered adduct ranges from $1.65 \AA$ Á to $1.99 \AA$ ̊ which is shorter than $\mathrm{O}-\mathrm{H}$ or $\mathrm{N}-\mathrm{H}$ covalent bond distances and thus characterizes hydrogen bonding interaction between CSDs and guanine. ${ }^{71}$ 


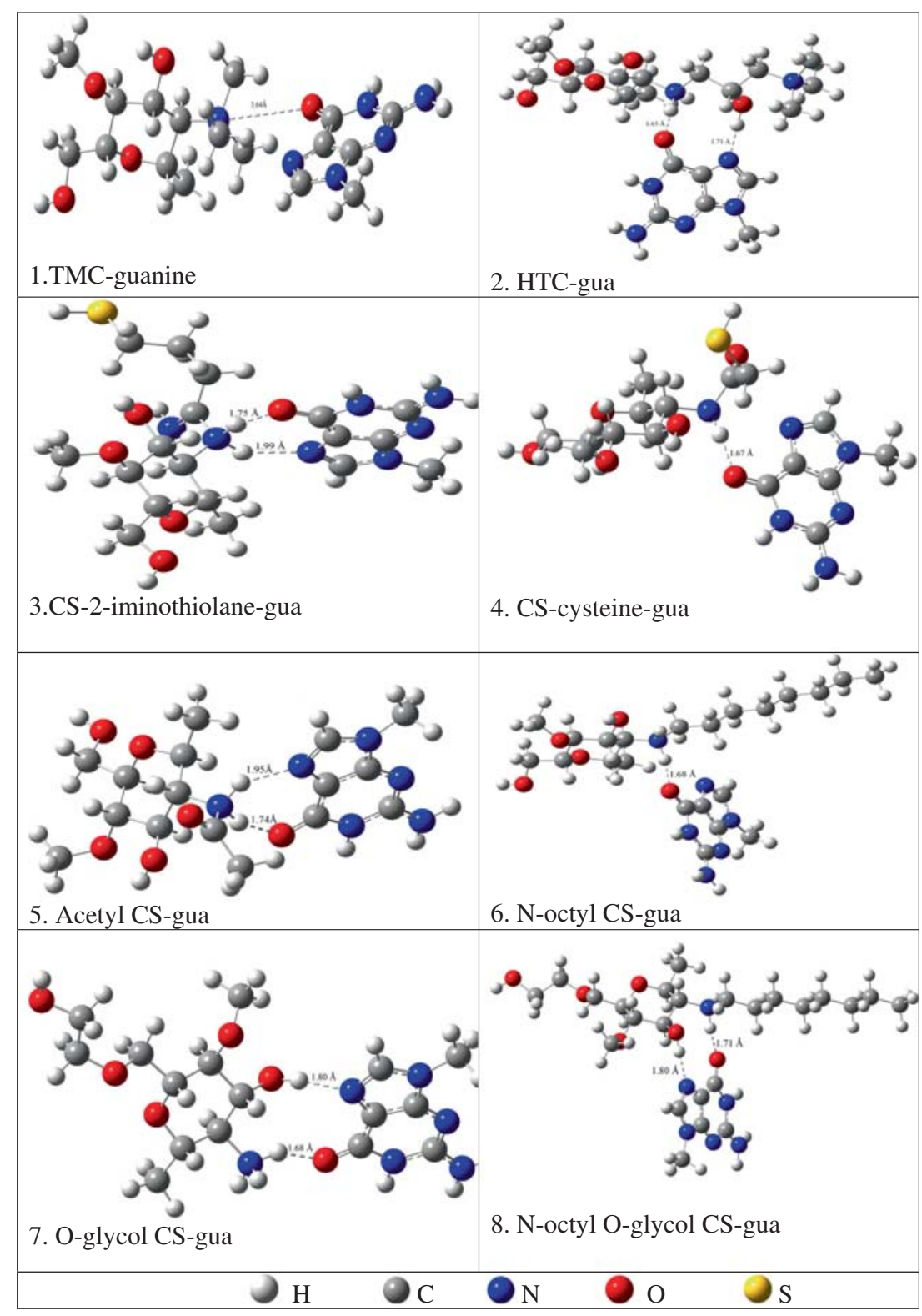

Figure 3. Optimized structure of CSD-gua adduct obtained at B3LYP 6-31G++(d,p) level of theory.

It further confirms the existence of hydrogen bonding between the two moieties in CSD-gua adducts which is also in good agreement with recent theoretical studies on hydrogen bond formation by chitosan and chitosan derivative. ${ }^{72,73}$ There exists two hydrogen bonds between CSD and guanine in the chosen adducts with the exceptions of CS-cysteine-gua (single), N-octyl CS-gua (single) and TMC-gua (nil).

TMC forms no hydrogen bond due to absence of any acidic $\mathrm{H}$ centre bonded to $\mathrm{N}$ atom of the amine group. It is interesting to note that the $\omega_{g}^{+}$value of the quarternary amine group in TMC (table 2) is higher than that of other CSDs and predicts a shorter $\mathrm{N}_{\mathrm{CS}}-\mathrm{O6}_{\text {gua }}$ distance than that observed in the optimized structure. Apart from increase in atomic radius in $\mathrm{N}-\mathrm{O}$ than $\mathrm{N}-\mathrm{H}$ or $\mathrm{O}-\mathrm{H}$, steric hindrance offered by three methyl groups attached to the $\mathrm{N}_{\mathrm{CS}}$ atom might play a role in comparatively longer $\mathrm{N}_{\mathrm{CS}}-\mathrm{O} 6_{\text {gua }}$ distance in TMC-gua adduct. Consequently, the weakest interaction with guanine is anticipated by TMC from among the chosen CSDs.

Two computational protocols are used to calculate the properties of a molecular system in the presence of solvents. The first one involves optimization of all the systems in gas phase and subsequent insertion of solvents during single-point calculation. The second one involves re-optimization of the systems in presence of solvents. In the present study, the second protocol is used; the geometrical minima of CSD-gua adducts are further ascertained in carbon tetrachloride, ethanol and in aqueous media. The observed $\mathrm{r}_{\mathrm{hb}}(\AA)$ ) between CSD and guanine in gas, $\mathrm{CCl}_{4}$ and aqueous media is presented in table 2. 
Table 2. $\mathrm{r}_{\mathrm{hb}}(\AA)$ ) between CSD amine hydrogen and N7and O6 atom of guanine.

\begin{tabular}{|c|c|c|c|c|}
\hline Adducts & Gas & $\mathrm{CCl}_{4}$ & Ethanol & Water \\
\hline TMC-gua & $\mathrm{N}-\mathrm{O}=3.64$ & $\mathrm{~N}-\mathrm{O}=3.71$ & $\mathrm{~N}-\mathrm{O}=3.69$ & $\mathrm{~N}-\mathrm{O}=3.69$ \\
\hline HTC-gua & $\begin{array}{l}\mathrm{H}-\mathrm{O}=1.65 \\
\mathrm{H}-\mathrm{N}=1.71\end{array}$ & $\begin{array}{l}\mathrm{H}-\mathrm{O}=1.67 \\
\mathrm{H}-\mathrm{N}=1.71\end{array}$ & $\begin{array}{l}\mathrm{H}-\mathrm{O}=1.69 \\
\mathrm{H}-\mathrm{N}=1.73\end{array}$ & $\begin{array}{l}\mathrm{H}-\mathrm{O}=1.69 \\
\mathrm{H}-\mathrm{N}=1.73\end{array}$ \\
\hline CS-2-iminothiolan-gua & $\begin{array}{l}\mathrm{H}-\mathrm{O}=1.75 \\
\mathrm{H}-\mathrm{N}=1.99\end{array}$ & $\begin{array}{l}\mathrm{H}-\mathrm{O}=1.74 \\
\mathrm{H}-\mathrm{N}=1.94\end{array}$ & $\begin{array}{l}\mathrm{H}-\mathrm{O}=1.74 \\
\mathrm{H}-\mathrm{N}=1.90\end{array}$ & $\begin{array}{l}\mathrm{H}-\mathrm{O}=1.74 \\
\mathrm{H}-\mathrm{N}=1.90\end{array}$ \\
\hline CS cysteine-gua & $\mathrm{H}-\mathrm{O}=1.67$ & $\mathrm{H}-\mathrm{O}=1.68$ & $\mathrm{H}-\mathrm{O}=1.69$ & $\mathrm{H}-\mathrm{O}=1.70$ \\
\hline Acetyl CS-gua & $\begin{array}{l}\mathrm{H}-\mathrm{O}=1.74 \\
\mathrm{H}-\mathrm{N}=1.95\end{array}$ & $\begin{array}{l}\mathrm{H}-\mathrm{O}=1.74 \\
\mathrm{H}-\mathrm{N}=1.9\end{array}$ & $\begin{array}{l}\mathrm{H}-\mathrm{O}=1.71 \\
\mathrm{H}-\mathrm{N}=1.85\end{array}$ & $\begin{array}{l}\mathrm{H}-\mathrm{O}=1.70 \\
\mathrm{H}-\mathrm{N}=1.82\end{array}$ \\
\hline N-octyl CS-gua & $\mathrm{H}-\mathrm{O}=1.68$ & $\mathrm{H}-\mathrm{O}=1.69$ & $\mathrm{H}-\mathrm{O}=1.73$ & $\mathrm{H}-\mathrm{O}=1.74$ \\
\hline O-glycol CS-gua & $\begin{array}{l}\mathrm{H}-\mathrm{O}=1.68 \\
\mathrm{H}-\mathrm{N}=1.80\end{array}$ & $\begin{array}{l}\mathrm{H}-\mathrm{O}=1.69 \\
\mathrm{H}-\mathrm{N}=1.79\end{array}$ & $\begin{array}{l}\mathrm{H}-\mathrm{O}=1.71 \\
\mathrm{H}-\mathrm{N}=1.76\end{array}$ & $\begin{array}{l}\mathrm{H}-\mathrm{O}=1.72 \\
\mathrm{H}-\mathrm{N}=1.75\end{array}$ \\
\hline N-octyl O-glycol CS-gua & $\begin{array}{l}\mathrm{H}-\mathrm{O}=1.71 \\
\mathrm{H}-\mathrm{N}=1.80\end{array}$ & $\begin{array}{l}\mathrm{H}-\mathrm{O}=1.71 \\
\mathrm{H}-\mathrm{N}=1.78\end{array}$ & $\begin{array}{l}\mathrm{H}-\mathrm{O}=1.70 \\
\mathrm{H}-\mathrm{N}=1.75\end{array}$ & $\begin{array}{l}\mathrm{H}-\mathrm{O}=1.70 \\
\mathrm{H}-\mathrm{N}=1.76\end{array}$ \\
\hline
\end{tabular}

Table 3. Interaction energy (in $\mathrm{kcal} / \mathrm{mol}$ ) of CS-gua and CSD-gua adduct.

\begin{tabular}{|c|c|c|c|c|}
\hline Adduct & $\Delta \mathrm{E}_{\text {int }}($ gas $)$ & $\Delta \mathrm{E}_{\text {int }}($ Carbon tetrachloride $)$ & $\Delta \mathrm{E}_{\text {int }}($ ethanol $)$ & $\Delta \mathrm{E}_{\text {int }}($ Water $)$ \\
\hline CS-GUA & -37.26 & -21.74 & -15.33 & -14.47 \\
\hline acetyl CS-gua & -41.49 & -26.15 & -18.76 & -17.77 \\
\hline CS- cysteine-gua & -36.77 & -21.81 & -14.71 & -13.73 \\
\hline CS-2iminothiolan-gua & -37.99 & -23.48 & -16.44 & -15.44 \\
\hline HTC-gua & -59.36 & -35.97 & -21.96 & -21.85 \\
\hline N-octyl CS-gua & -33.98 & -19.36 & -12.10 & -11.09 \\
\hline N-octyl O-glycol CS-gua & -34.67 & -21.36 & -15.88 & -15.04 \\
\hline O-glycol CS-gua & -43.75 & -35.53 & -15.47 & -14.60 \\
\hline TMC-gua & -23.62 & -9.78 & -4.58 & -3.82 \\
\hline
\end{tabular}

Lengthening in $\mathrm{r}_{\mathrm{hb}}(\AA)$ ) in solvent media is less than $0.1 \AA ̊$ in all the considered adducts (table 2). A similar marginal effect of solvation on hydrogen bond length (variation of around $0.11 \AA$ Á) was reported by Hwang and Chung. ${ }^{74}$

A secondary interaction such as hydrogen bonding was demonstrated to be responsible for the binding between CS and nucleobases. ${ }^{22,23}$ The present study establishes the presence of hydrogen bonding in protonated CSD-guanine interactions.

\subsection{Interaction energy $\left(\Delta E_{\text {int }}\right)$}

The magnitude of interaction energy of CSDnucleobase adduct is crucial in terms of the protection of complexed DNA from degradation in the course of shuttling a DNA payload into the cell as well as the transfer of DNA near or within the nucleus of a target cell. A high value of $\Delta \mathrm{E}_{\text {int }}$ favours strong binding between the two moieties in adduct while decrease in $\Delta \mathrm{E}_{\text {int }}$ facilitates dissociation of adduct. The values of $\Delta \mathrm{E}_{\text {int }}$ obtained for different adducts are presented in table 3 .
Gas phase $\Delta \mathrm{E}_{\mathrm{int}}$ value is the highest in HTC-gua among all the considered adducts which may be attributed to greater positive charge $(+2)$ of the entity. As anticipated from bond length values, the lowest $\Delta \mathrm{E}_{\text {int }}$ is observed in case of TMC-gua.

A steady drop in $\Delta \mathrm{E}_{\text {int }}$ with increasing polarity of solvent medium is observed in all the chosen adducts. This is in conformity with the trend expected from group philicity values of amine group in CSDs (table 1). The extent of lowering in $\Delta \mathrm{E}_{\text {int }}$ values in aqueous phase from that in gas phase is similar to that observed in case of interaction between positively charged drug intermediate and GC base pair. ${ }^{75}$ Results thus advocate a strong interaction between the CSDs and nucleobases in non-polar medium and gradual weakening of the interaction as the CSD-DNA complex traverses through the cell membrane (protein-lipid bilayer, which is non-polar in nature). In cytoplasm (which is polar in nature) the interaction is thus expected to be weakest that might facilitate dissociation of the two moieties. Very low $\Delta \mathrm{E}_{\text {int }}$ for TMC-gua $(-2.33 \mathrm{kcal} / \mathrm{mol})$ in aqueous phase indicates greater suitability of TMC as a gene carrier which is in qualitative agreement with earlier literature. ${ }^{15}$ 
It is interesting to observe that solvent phase $\Delta E_{\text {int }}$ values do not comply with the general BE-BL relationship [which states that for molecular structures with the same type of atoms in their local minima, and free from any strain, the shorter the bond length (BL), the higher the bond energy $(\mathrm{BE})]$. The hydrogen bond length values virtually unaffected by varying polarity of solvent, suggests a similar trend in case of $\Delta \mathrm{E}_{\text {int }}$ also. On the contrary, a steady drop in $\Delta \mathrm{E}_{\text {int }}$ with increasing polarity is observed. Cina et al., have explained such behavior in presence of an external electric field. ${ }^{76}$ The present study suggests a similar breaking of BE-BL principle by CSD-guanine interaction in solvent media.

\subsection{Stability of CSD-gua adducts}

3.4a Study of global hardness of CSD-gua adducts: With an aim to estimate the chemical stability of the CSD-gua adducts during their transfection, we have calculated global hardness of adducts CS-gua and CSD-gua in gas phase as well as in carbon tetrachloride ethanol and aqueous medium. A comparison in global hardness values of the chosen adducts is presented in figure 4.

Adducts namely CS-gua, CS-cysteine-gua, acetyl CSgua, O-glycol CS-gua, TMC-gua, $N$-octyl O-glycol CS-gua, and N-octyl CS-gua possess almost similar gas phase global hardness which is around $50 \mathrm{kcal} / \mathrm{mol}$ and $20 \mathrm{kcal} / \mathrm{mol}$ higher than that of HTC-gua and CS2-iminothiolan-gua respectively. Results indicate that highly cationic HTC-gua is most vulnerable for cellular degradation. Application of solvent phase does not affect global hardness of adducts except in case of HTC and CS-2-iminothiolane-gua; wherein a marginal increase in global hardness with increasing polarity of solvents is noticed. This result indicates that except HTC-gua and CS-2-iminothiolane-gua, chemical stability of CSD-gua adducts remains insensitive to the nature of prevailing media. Inclusion of solvent effect shows that trends of reactivity in terms of both global and local descriptors is different in solvent phase with respect to their trend in the gas phase, which is consistent with earlier literature. ${ }^{65}$

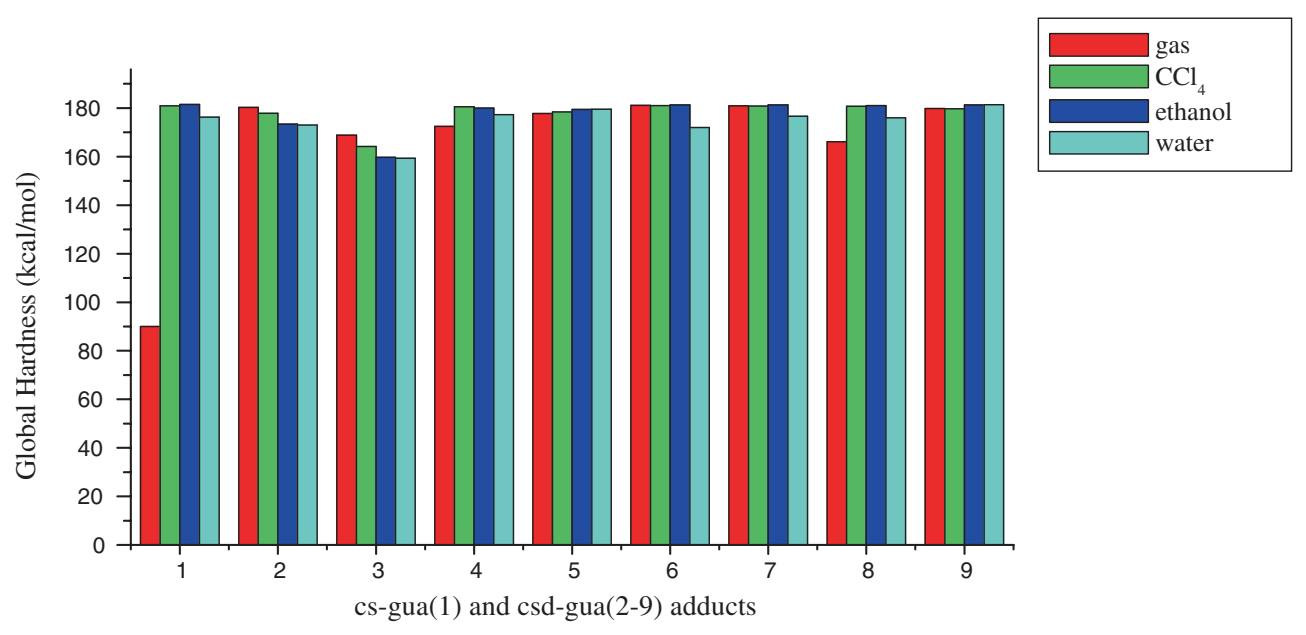

Figure 4. Global Hardness (in $\mathrm{kcal} / \mathrm{mol}$ ) of the chosen adducts in different medium at CAMB3LYP 6-31G++(d,p) level of theory. 1 HTC-gua, 2 CS-2-iminothiolane-gua, 3 CS-cysteine-gua, 4 acetyl CS-gua, 5 CS-gua, 6 N-octyl O-glycol CS-gua, 7 N-octyl CS-gua, 8 TMC-gua, and 9 O-glycol CS-gua.

Table 4. $\Delta \mathrm{G}_{\mathrm{sol}}$ values of CSD-gua adducts (in $\mathrm{kcal} / \mathrm{mol}$ ).

\begin{tabular}{lccc}
\hline Adduct & $\begin{array}{c}\Delta \mathrm{G}_{\text {sol }} \text { (carbon } \\
\text { tetrachloride) }\end{array}$ & $\Delta \mathrm{G}_{\text {sol }}$ (ethanol) & $\Delta \mathrm{G}_{\text {sol }}$ (water) \\
\hline CS-gua & -46.00 & -76.96 & -72.69 \\
acetyl CS-gua & -44.20 & -70.94 & -65.54 \\
CS-cysteine- gua & -44.92 & -73.34 & -67.22 \\
CS-2-iminothiolane-gua & -46.45 & -75.32 & -68.54 \\
HTC-gua & -99.87 & -167.37 & -161.29 \\
N-octyl CS-gua & -46.51 & -71.51 & -60.92 \\
N-octyl O-glycol CS-gua & -50.00 & -77.91 & -67.32 \\
O-glycol CS-gua & -41.18 & -73.70 & -69.03 \\
TMC-gua & -42.70 & -68.77 & -19.43 \\
\hline
\end{tabular}


3.4b Free energy of solvation $\left(\Delta G_{\text {sol }}\right)$ of CSD-gua adduct: Thermodynamic stability of adduct in solvent media can be gauged by free energy of solvation which is also indicative of its extent of solubility. Calculated $\Delta \mathrm{G}_{\text {sol }}$ values for different adducts are presented in table 4 .

Abnormally high $\Delta \mathrm{G}_{\text {sol }}$ value of HTC-gua is apparently due to the higher charge $(+2)$ of the adduct which attracts a large scale of solvation and this data further substantiates the highest $\Delta \mathrm{E}_{\text {int }}$ value associated with HTC-gua adduct in table 3. Calculated values suggest greater thermodynamic stability of adducts in polar media and also agree with results of earlier relevant studies. ${ }^{65,73}$ Notably, TMC-gua is thermodynamically most stable in carbon tetrachloride phase and least stable in aqueous phase. This result suggests that in nonpolar protein-lipid bi-layer of cell membrane, TMC-gua sustains best while in polar cytoplasmic medium its dissociation is greatly favoured, an observation that complies with earlier comment of greater suitability of TMC as DNA carrier.

\section{Conclusions}

The prime object of this study is to provide an insight into the reactivity of a few CSDs and their interaction with nucleobases in DNA. Eight CSDs covering different mode of functionalization were chosen for the study. Our study confirms the presence of hydrogen bonding between positively charged amine group of CSDs and hetero atoms of nucleobase (guanine as a representative case in this study). Reactivity of CSDs and strength of their interaction with nucleobases, both are sensitive to the nature of functional modification as well as to the prevailing media. All the chosen derivatives exhibit stronger interaction in non-polar medium and there is a gradual weakening with increasing polarity of the medium. Chemical stability of CSD-gua adducts is unaffected by changing media while thermodynamically the considered adducts are less stable in polar solvent. Most of the considered CSD-gua adducts exhibit comparable stability with CS-gua adduct. In addition, stronger interaction between CSDs and guanine in nonpolar medium and a gradual weakening with increasing polarity of medium predicts improved efficiency of the derivatives than that of unmodified CS as a DNA carrier. In addition, among the chosen derivatives, TMC shows greater suitability as a DNA carrier.

Despite the promises shown by chitosan as a gene carrier and the enormous effort put in developing efficient chitosan-based vectors for gene delivery, the therapeutic effectiveness of chitosan based gene therapy still needs to be improved in order to achieve clinical significance. A thorough understanding of various aspects pertinent to the binding and transferring of NA by CS derivatives is envisaged to play an important role as predictive tool in formulating chitosan aided gene delivery system. This study is believed to boost future research in designing of nucleic acid carriers based on chitosan derivatives with new insights.

\section{Acknowledgements}

Authors thank DST, New Delhi for financial support (Grant No. SERB/F/5846/2014-15).

\section{References}

1. MacLaughlin F C, Mumper R J, Wang J, Tagliaferri J M, Gill I, Hinchcliffe M and Rolland A P 1998 J. Cont. Rel. 56259

2. Lee K Y, Kwon I C, Kim Y H and Jeong S Y $1998 J$. Cont. Rel. $\mathbf{5 1} 213$

3. Shu X Z and Zhu K J 2002 Eur. J. Pharm. Biopharm. 54 235

4. Lee M K, Shun S K, Choi W J, Kim J K, Choi S H, Kim A, Oungbho K, Park J S, Ahn W S and Kim C K 2005 Biomaterials 262147

5. Schnürch A B and Dünnhaupt S 2012 Eur. J. Pharm. Biopharm. 81463

6. Richardson S C W, Kolbe H V J and Duncan R 1999 Int. J. Pharm. 178231

7. Park I K, Kim T H, Kim S I, Park Y H, Kim W J, Akaike T and Cho C S 2003 Int. J. Pharm. 257103

8. Mourya V K and Inamdar N N 2008 React. Funct. Polym. 681013

9. Prabaharan M and Tiwari A 2011 In Chitin, Chitosan, Oligosaccharides and Their Derivatives in Biological Activities and Applications Se-Kwon Kim (Ed.) (Boca Raton: CRC Press) p. 173

10. Thanou M M and Kotze A F 2000 J. Cont. Rel. 6415

11. van der Merwe S M, Verhoef J C, Verheijden J H M, Kotzé A F and Junginger H E 2004 Eur. J. Pharm. Biopharm. 58225

12. Tien Le, Lacroix C M, Ispas-Szabo $P$ and Mateescu M A 2003 J. Cont. Rel. 931

13. Federica S and Bernkop-Schnürch A 2011 In Chitosan for Biomaterials I R Jayakumar M Prabaharan and Riccardo A A Muzzarelli (Eds). Advances in Polymer Science Vol. 243 (Springer-Verlag Berlin: Heidelberg) pp. $93-110$

14. Liu W G, Yao K D and Liu Q G 2001 J. Appl. Polym. Sci. 823391

15. Liu W G, Zhang X, Sun S J, Sun G J, Yao K D and Liang D C 2003 Bioconjugate Chem. 14782

16. Thongchai S and Boonmee J 2011 Int. J. Mol. Sci. 12 1672

17. Kim J H, Kim Y S, Kim S W, Park J H, Kim K, Choi K, Chung H, Jeong S Y, Park R W, Kim I S and Kwon I C 2006 J. Cont. Rel. 111228 
18. Park J H, Kwon S, Lee M, Chung H, Kim J H, Kim Y S, Park R W, Kim I S, Seo S B, Kwon I C and Jeong S Y 2006 Biomaterials 27119

19. Lee E S, Park K H, Park I S and Na K 2007 Int. J. Pharm. 338310

20. Pack D, Hoffman A S, Pun S and Stayton P S 2005 Nat. Rev. Drug Discov. 4581

21. Read M L, Logan A and Seymour L 2005 Adv. Genet. 5319

22. Mao S, Sun W and Kissel T 2010 Adv. Drug Del. Rev. 6212

23. Deka B C and Bhattacharyya P K 2015 Comput. Theor. Chem. 105135

24. Kohn W, Becke A D and Parr R G 1996 J. Phys. Chem. 10012974

25. Baerends E J and Gritsenko O V 1997 J. Phys. Chem. A 105383

26. Chermette H 1998 Coord. Chem. Rev. 178699

27. Andrews L and Citra A 2002 Chem. Rev. 102885

28. Ziegler T and Autschbach J 2005 Chem. Rev. 1052695

29. Neese F 2009 Coord. Chem. Rev. 253526

30. Schultz N E, Zhao Y and Truhlar D G 2005 J. Phys. Chem. A 10911127

31. Beranek D T 1990 Mutat. Res. 23111

32. Singer B and Grunberger D 1983 In Molecular biology of mutagens and carcinogens (New York: Plenum)

33. Brookes P and Lawley P D 1963 Biochem. J. 89138

34. Bhattacharyya P K, Sinha S, Sarmah N and Deka B C 2015 In Frontiers in Computational Chemistry Vol. 2, $1^{\text {st }}$ edition, Zaheer-ul-Haq and J D Madura (Eds.) (UAE: Bentham Science Publishing House) p. 121

35. Deka B C and Bhattacharyya P K 2016 Comput. Theor. Chem. 107872

36. Soniat M, Rogers D M and Rempe S B 2015 J. Chem. Theory Comput. 112958

37. DiLabio G A, Johnson E R and de la Roza A O 2013 Phys. Chem. Chem. Phys. 1512821

38. Becke A D 1993 J. Chem. Phys. 985648

39. Lee C, Yang W and Parr R G 1988 Phys. Rev. B 37785

40. Parr R G and Yang W 1989 In Density Functional Theory of Atoms and Molecules (Oxford University Press: New York)

41. Parthasarathi R, Padmanabhan J, Elango M, Subramanium V and Chattaraj P K 2004 Chem. Phys. Lett. 394 225

42. Chandra A K and Nguyen M T 2007 Faraday Discuss 135191

43. Molteni G and Ponti A 2003 Chem. Eur. J. 92770

44. Nguyen H M T, Peeters J, Nguyen M T and Chandra A K 2004 J. Phys. Chem. A 108484

45. Roy R K 2003 J. Phys. Chem. A 107397

46. Melin J, Aparicio F, Subramanian V, Galvan M and Chattaraj P K 2004 J. Phys. Chem. A 1082487

47. Geerlings P, Proft F D and Langenaekar W 2003 Chem. Rev. 1031793

48. Kar R and Pal S 2010 Int. J. Quant. Chem. 1101642

49. Parr R G and Pearson R G 1983 J. Am. Chem. Soc. 105 7512

50. Parr R G, Donnelly R A, Levy M and Palke W E 1978 J. Chem. Phys. 683801

51. Koopmans T 1934 Physica 1104

52. Parr R G, Szentpaly L V and Liu S 1999 J. Am. Chem. Soc. 1211922
53. Chandrakumar K R S and Paul S 2002 Int. J. Mol. Sci. 3 324

54. Hirshfeld F L 1977 Theoret. Chim. Acta. 44129

55. Roy R K, Pal S and Hirao K 1999 J. Chem. Phys. 110 8236

56. Roy R K, Hirao K and Pal S 2000 J. Chem. Phys. 113 1372

57. Padnabhan J, Parthasarathi R, Subramanium V and Chattaraj P K 2006 Chem. Res. Toxicol. 19356

58. Galano A and Idaboy J R A 2006 J. Comput. Chem. 27 1203

59. Tomasi J and Persico M 1994 Chem. Rev. 942027

60. Tomasi J, Mennucci B and Cammi R 2005 Chem. Rev. 1052999

61. Marenich V, Cramer C J and Truhlar D G 2009 J. Phys. Chem. B 1136378

62. Frisch M J, Trucks G W, Schlegel H B, Scuseria G E, Robb M A, Cheeseman J R, Scalmani G, Barone V, Mennucci B, Petersson G A, Nakatsuji H, Caricato M, Li X, Hratchian H P, Izmaylov A F, Bloino J, Zheng G, Sonnenberg J L, Hada M, Ehara M, Toyota K, Fukuda R, Hasegawa J, Ishida M, Nakajima T, Honda Y, Kitao O, Nakai T, Vreven J A, Montgomery Jr, Peralta J E, Ogliaro F, Bearpark M, Heyd J J, Brothers E, Kudin K N, Staroverov V N, Kobayashi R, Normand J, Raghavachari K, Rendell A, Burant J C, Iyengar S S, Tomasi J, Cossi M, Rega N, Millam J M, Klene M, Knox J E, Cross J B, Bakken V, Adamo C, Jaramillo J, Gomperts R, Stratmann R E, Yazyev O, Austin A J, Cammi R, Pomelli C, Ochterski J W, Martin R L, Morokuma K, Zakrzewski V G, Voth G A, Salvador P, Dannenberg J J, Dapprich S, Daniels A D, Farkas Ö., Foresman J B, Ortiz J V, Cioslowski J and Fox R A D J. 2009 Gaussian 09, Gaussian Inc., Wallingford, CT; 2010 Revision B.01, (Gaussian, Inc.: Wallingford CT)

63. Parr R G and Chattaraj P K 1991 J. Am. Chem. Soc. 113 1854

64. Neog B, Sinha S and Bhattacharyya P K 2013 Comput. Theor. Chem. 101819

65. Sharma P and Deka R C 2008 Int. J. Quant. Chem. 108 1400

66. Chattaraj P K, Maiti B and Sarkar U 2003 J. Phys. Chem. 1074973

67. Padmanabhan J, Parthasarathi R, Sarkar U, Subramanian V and Chattaraj P K 2004 Chem. Phys. Lett. 383 122

68. Leworasirikul A, Yokyama S, Noguchi K, Ogawa K and Okuyama K 2004 Carbohydr. Res. 339825

69. Ogawa K, Yui T and Okuyama K 2004 Int. J. Biol. Macromol. 341

70. Siraleartmukul K, Siriwong K, Remsungnen T, Muangsin N, Udomkichdecha W and Hannongbua S 2004 Chem. Phys. Lett. 395233

71. Gilli P and Gilli G 2009 In The Nature of the Hydrogen Bond (Oxford: Oxford University Press)

72. Srinophakun T and Boonmee J 2011 Int. J. Mol. Sci. 12 1672

73. Pal S and Kundu T K 2013 Chem. Sci. Trans. 2447

74. Hwang S and Chung D S 2005 Bull. Korean Chem. Soc. 26589

75. Neog B, Sarmah N, Kar R and Bhattacharyya P K 2012 Comput. Theor. Chem. 98679

76. Cina F-N, Novák M and Marek R 2013 J. Phys. Chem. C 11721509 